\title{
Evaluating the Accuracy of Mobile Digital Shade Matching Application andits Comparison with Reflectance Spectrophotometer System for All Ceramic and Metal Ceramic Restoration
}

\author{
Siddhesh Borse*, Sachin Chaware and Bikash Pattnaik \\ Department of Prosthodontics and Crown and Bridge, MGV KBH Dental College \\ and Hospital Nashik, India \\ *Corresponding Author: Siddhesh Borse, Department of Prosthodontics and \\ Crown and Bridge, MGV KBH Dental College and Hospital Nashik, India.
}

Received: October 11, 2021

Published: October 29, 2021

(C) All rights are reserved by Siddhesh Borse., et al.

\begin{abstract}
Purpose: The purpose of the study was to evaluate the accuracy mobile digital shade matching application and its comparison with spectrophotometer system for all ceramic and metal ceramic restoration.

Methods: This study was conducted on 10 subjects. Patients visiting the outpatient department has been selected for the study. Samples has been divided into 3 groups: Group I: (Control group) Visual Shade Guide, Group II: (Test group) Mobile Digital Shade Matching Application, Group III: Spectrophotometer

Results: Visual shade method observed highest $\Delta \mathrm{E}$ value. $\Delta \mathrm{E}$ value was lowest for All-ceramic crowns recorded by spectrophotometer and lowest $\Delta \mathrm{E}$ value for mobile digital application for Metal-ceramic crowns. Equal clinical acceptance for crowns fabricated by mobile application and spectrophotometer. However, lowest clinical acceptance for visual shade method.

Conclusion: Mobile digital application may be the emerging method for shade selection, but further studies required to define its role in shade selection.
\end{abstract}

Keywords: Visual Shade; Vita Easy Shade Spectrophotometer; Mobile Application

\section{Introduction}

Dental practitioner must have the understanding about the color science and its properties while using the restorative material such as ceramic, composite, and acrylic resin material [1]. Color determination in dentistry can be divided into two categories: Visual and Instrumental shade selection [2].

Visual shade selection uses a series of shade tabs in the sequence order in relation to the tooth shade. The benefits of visual shade guide system are that it is simple, economical and convenient to use for dental practitioner as well as dental technician [3]. The subjective error is the variation in evaluation between two practitioner (intra-practitioner) or the practitioner and dental techni- cian $[4,5]$. These variations may be due to the metamerism $[2,6]$ and it is further affected due to color fatigue by observing one color for long time, aging, chronic illness, glaucoma, color blindness and medication like antiepileptic drugs affect the visual acuity $[7,8]$.

Instrumental methods shade analysis has reported more accurate and more consistent compared with human shade assessment. The advantages are no influence of surroundings or lighting and the results being reproducible $[9,10]$. The instrumental method is categorised in RGB devices, Colorimeters, Digital camera and Spectrophotometers. Instrumental method produces better shade matching compared to visual shade matching, since it eliminates the subjective error. However, instrumental method records only 
one tooth shade matching and shade selection were dissimilar for curved and flat tooth surface Present study develops the mobile shade application and compared with the spectrophotometer. Study has evaluated the pilot result.

\section{Material and Methods}

Present study is a pilot comparative observational study that performs the analysis of tooth shade between Mobile digital shade matching application and spectrophotometer. The subjects were selected randomly from the outpatient department, between the age group of 20 to 40 years of either sex and rendering informed consent through signed document to participate in the study over a study period of 18 months baseline. This study was conducted on 10 subjects between the age group of 20-40 years from outpatient department. Maxillary central incisor was selected as a reference tooth with no loss tooth structure and minimal discoloration. A total 10 patients were selected for pilot study. Out of that 5 were subjected for metal ceramic crown and remaining 5 for all ceramic crown. Similarly, 30 crowns were fabricated, out of that 15 were metal ceramic rest of the 15 were all ceramic. Three crowns were fabricated for each patient as per the three shades selection method were used for the study.

- Group I: (Control group) Visual Shade Guide (vita classical shade tabs)

- Group II: (Test group) Mobile Digital Shade Matching Application

- Group III: Reflectance Spectrophotometer (VITA Easyshade V)

\section{Inclusion criteria}

- $\quad$ Subjects were selected within age group of 20 to 40 years of either sex.

- Maxillary central incisor was selected as it has maximum tooth circumference.

- Selected tooth was either vital or non-vital provided that there was minimal loss of tooth structure.

\section{Exclusion criteria}

- Subjects with age group less than 20 years and more than 40 years have not been included in study.

- Selected abutment tooth other than maxillary central incisor was not included in study.

- There was no periodontal disease, gingival recession and mobility to the selected abutment tooth.

\section{Tooth shade selection}

Shade evaluation was completed prior to tooth preparation. The shade was selected once before tooth preparation by using Vita classical shade guide (VITA Zahnfabrik, Bad Sackingen, Germany) Mobile Digital Shade Matching Application, and Vita easyshade V (VITA Zahnfabrik, Bad Sackingen, Germany)

Shade selection was performed by the single investigator and manufacturing instruction were followed. Android mobile digital application was used for shade selection. Mobile application was based upon $\mathrm{L}^{*} \mathrm{a}^{*} \mathrm{~b}^{*}$ values. While taking the tooth image mobile was held perpendicular to the tooth surface to eliminate the surrounding light for better tooth image.

\section{Results}

Since, it was a pilot study with minimal sample size statistical analysis was not performed. But, the result was evaluated by using $\Delta \mathrm{E}$ value. Highest $\Delta \mathrm{E}$ value was recorded by the visual method followed by mobile digital application and lowest by spectrophotometer in all ceramic crown. However, in metal ceramic group reported lowest $\Delta \mathrm{E}$ value by mobile digital application. There is a marginal difference of $\Delta \mathrm{E}$ value between spectrophotometer and mobile digital application. The observation of investigator and operator reported maximum acceptance for mobile digital application and spectrophotometer of shade comparison between ceramic crown and reference tooth. On overall comparison using Pearson Chi square test, statistically significant $(\mathrm{p}<0.05)$ difference among three groups for patient acceptance. On pairwise comparison, there was observed highly statistically significant ( $p$ $<0.001$ ) difference for Group I with Group II and Group III for patient acceptance. Statistically significant $(p<0.05)$ was observed between Group II and III. Visual shade recorded the lowest acceptance.

\section{Discussion}

There are several mobile applications available on google play store, most of the application were product based advertise application. The present mobile application is completely for shade evaluation. Hence, the aim of the present study to compare the shade evaluation among mobile digital application and spectrophotometer. Spectrophotometer as selected because it is more reliable instrument for shade evaluation. Several studies reported that spectrophotometer produces more accurate shade than visual shade selection. Spectrophotometer also recorded the $\Delta \mathrm{E}$ value for the present study. VITA classical shade guide was selected for shade evaluation because it produces better shade matching then other commercial shade tabs.

Result of the study reported that $\Delta \mathrm{E}$ value is the most accurate measurement of color difference between two objects. Present study showed lowest $\Delta \mathrm{E}$ value for mobile digital application for metal ceramic group followed by spectrophotometer. Whereas, all 
ceramic group observed lowest $\Delta \mathrm{E}$ value for spectrophotometer However, there was a slight difference of $\Delta \mathrm{E}$ value between mobile application and spectrophotometer which was clinically not significant. Visual shade method showed highest $\Delta \mathrm{E}$ value $(>2)$ which was clinically not acceptable.

A total 10 patients were selected for pilot study. Out of that 5 were subjected for metal ceramic crown and remaining 5 for all ceramic crown. Similarly, 30 crowns were fabricated, out of that 15 were metal ceramic rest of the 15 were all ceramic. Three crowns were fabricated for each patient as per the three shade selection method were used for the study. The observation between the investigator and operator reported maximum crown rejection for visual shade guide method. Most of the crown of visual shade method show highest shade difference compared to adjacent central incisor. Apart from the shade difference, crowns were also rejected due to faulty contour and bulky appearance. However, in mobile digital application observed maximum acceptance for metal ceramic group and there was maximum acceptance of spectrophotometer for all ceramic group. But there was not much clinical significance between mobile application and spectrophotometer, as crown of both methods were accepted or rejected due to slight difference.

\section{Conclusion}

The present study concluded that instrumental method is much superior then visual method. Mobile digital application may be newly emerging technique for shade evaluation but further study is needed to evaluate its precise role. The limitation of the present study that it was only performed on single tooth. Hence, further clinical evaluation of multiple anterior restoration.

\section{Bibliography}

1. Chu SJ. "Fundamentals of Color: Shade Matching and Communication in Esthetic Dentistry". Quitessence Publishing Co. Inc (2004).

2. Sikri VK. "Color: Implications in dentistry". Journal of Conservative Dentistry 13.4 (2010): 249-255.

3. Parameswaran V., et al. "Comparison of accuracies of an intraoral spectrophotometer and conventional visual method for shade matching using two shade guide systems". Journal of Indian Prosthodontic Society 16.4 (2016): 352.

4. McLaren EA. "Shade Analysis and Communication". Inside Dentistry 6.5 (2010): 58-66.

5. Bergen SF and McCasland J. "Dental operatory lighting and tooth color discrimination". Journal of the American Dental As sociation 94.1 (1977): 130-134.
6. Baltzer A and Jinoian VK. "Determination of tooth colors". Quintessenz Zahntech 30 (2004): 726-740.

7. Berns RS. "Billmeyer and Saltzman's principles of color technology". John Wiley and Sons (2019).

8. Wyszecki G and Stiles WS. "Color science: concepts and methods, quantitative data and formulas". New York: John Wiley (1982): 83-173.

9. Hasegawa A., et al. "Color and translucency of in vivo natural central incisors". Journal of Prosthetic Dentistry 83.4 (2000): 418-423.

10. Marcucci B. "Using tooth and color guides together". Journal of Prosthetic Dentistry 86.3 (2001): 322-323.

Volume 5 Issue 11 November 2021

(C)All rights are reserved by Siddhesh Borse., et al. 